\title{
Numerical Analyses of Discrete Gust Response for an Aircraft
}

\author{
Guowei Yang* \\ Chinese Academy of Sciences, 100080 Beijing, People's Republic of China \\ and \\ Shigeru Obayashi ${ }^{\dagger}$ \\ Tohoku University, 980-8577 Sendai, Japan
}

\begin{abstract}
Based on Navier-Stokes equations and structural and flight dynamic equations of motion, dynamic responses in vertical discrete gust flow perturbation are investigated for a supersonic transport model. A tightly coupled method was developed by subiterations between aerodynamic equations and dynamic equations of motion. First, under the assumption of rigid-body and single freedom of motion in the vertical plunging, the results of a direct-coupling method are compared with the results of quasi-steady model method. Then, gust responses for the one-minus-cosine gust profile are analyzed with two freedoms of motion in plunging and pitching for the airplane configurations with and without the consideration of structural deformation.
\end{abstract}

\section{Introduction}

G UST load is one of the important dynamic loads considered in aircraft structure design. Because of its multidisciplinary nature with aerodynamics, flight dynamics, aeroelasticity, and atmospheric turbulence, until now, only the doublet-lattice, unsteady linear aerodynamic code DLM, coupled with the equation of motion of flexible vehicle, was used for gust response analysis. ${ }^{1-3}$

Gusts in nature tend to be random. The early design methods for gust loads were based on a single discrete gust having a oneminus-cosine velocity profile. Recently, the statistical discrete gust method and the power spectral density method ${ }^{4}$ in the frequency domain have been used to define the gust loads, however, which are still hard to combine with the modern Navier-Stokes numerical method.

In recent years, for the motion of rigid vehicles, the path of stores during the separation phase has mainly been investigated with the computational fluid dynamics (CFD) algorithm coupled with a sixdegree-of-freedom (6DOF) algorithm..$^{5-8}$ For the motion of a flexible body, only Leishman and his team at the University of Maryland did this kind of work for the rotor blades. Insofar as the authors know, the computation of gust response with the coupling method has still not been reported. In this paper, the fully implicit multiblock Navier-Stokes aeroelastic solver implemented by Yang et al., ${ }^{9}$ coupled with the flight and structural dynamic equations of motion, has been developed to simulate gust dynamic responses, which can model the motion of rigid or flexible vehicles. Because it is hard to find the structural data of a flexible vehicle, the supersonic transport (SST) designed by National Aerospace Laboratory of Japan (NAL) ${ }^{10}$ which has high rigidity, is taken as our calculated case. To study the effects of dynamic response due to flow perturbation and airplane motion, which involves only the consideration of vertical plunging motion, a comparative study was first done for the airplane in the harmonic flow perturbation with the direct-coupling method and the quasi-steady model method. Then, the gust responses in a one-minus-cosine gust velocity profile are analyzed with two freedoms of motion in plunging and pitching, with and without the consideration of structural deformation.

Received 18 May 2003; presented as Paper 2003-3513 at the 21st Applied Aerodynamics Conference, Orlando, FL, 23 June 2003; revision received 7 February 2004; accepted for publication 9 February 2004. Copyright (C) 2004 by the American Institute of Aeronautics and Astronautics, Inc. All rights reserved. Copies of this paper may be made for personal or internal use, on condition that the copier pay the $\$ 10.00$ per-copy fee to the Copyright Clearance Center, Inc., 222 Rosewood Drive, Danvers, MA 01923; include the code 0021-8669/04 \$10.00 in correspondence with the CCC.

*Professor, Key Laboratory of High-Temperature Gas Dynamics, Institute of Mechanics.

${ }^{\dagger}$ Professor, Institute of Fluid Science. Associate Fellow AIAA.

\section{Aerodynamic Equations and Numerical Method}

Aerodynamic governing equations are the unsteady, threedimensional, thin-layer Navier-Stokes equations in strong conservation law form, which can be written in curvilinear coordinates as

$$
\partial_{t} \hat{Q}+\partial_{\xi} F+\partial_{\eta} G+\partial_{\zeta} H=\partial_{\zeta} H_{v}+S_{\mathrm{GCL}}
$$

The source term $S_{\mathrm{GCL}}$ is obtained from the geometric conservation law (GCL) for a moving mesh. ${ }^{11}$ In the formulation, all variables are normalized by the appropriate combination of freestream density, freestream velocity, and mean aerodynamic chord length. The viscosity coefficient $\mu$ in $H_{v}$ is computed as the sum of laminar and turbulent viscosity coefficients, which are evaluated by Sutherland's law and the Baldwin-Lomax model. ${ }^{12}$

The lower-upper symmetric Gauss-Seidel (LU-SGS) method (see Ref. 13), employing a Newton-like subiteration, is used for the solution of Eq. (1). Second-order temporal accuracy is obtained by utilization of a three-point backward difference in the subiteration procedure. The numerical algorithm can be deduced as

$$
\begin{aligned}
& L D^{-1} U \Delta Q=-\phi^{i}\left\{(1+\phi) Q^{p}-(1+2 \phi) Q^{n}+\phi Q^{n-1}\right. \\
& \left.\quad-J \Delta t Q^{p} S_{\mathrm{GCL}}^{p}+J \Delta t\left[\delta_{\xi} F^{p}+\delta_{\eta} G^{p}+\delta_{\zeta}\left(H^{p}-H_{v}^{p}\right)\right]\right\}
\end{aligned}
$$

where

$$
\begin{gathered}
L=\bar{\rho} I+\phi^{i} J \Delta t\left(A_{i-1, j, k}^{+}+B_{i, j-1, k}^{+}+C_{i, j, k-1}^{+}\right), \quad D=\bar{\rho} I \\
U=\bar{\rho} I-\phi^{i} J \Delta t\left(A_{i+1, j, k}^{-}+B_{i, j+1, k}^{-}+C_{i, j, k+1}^{-}\right) \\
\bar{\rho}=1+\phi^{i} J \Delta t[\bar{\rho}(A)+\bar{\rho}(B)+\bar{\rho}(C)], \quad \phi^{i}=1 /(1+\phi) \\
\Delta Q=Q^{p+1}-Q^{p}
\end{gathered}
$$

Here, $\phi=0.5$, and $Q^{p}$ is the subiteration approximation to $Q^{n+1}$. As $p \rightarrow \infty, Q^{p} \rightarrow Q^{n+1}$. The deduced subiteration scheme reverts to the standard first-order LU-SGS scheme for $\phi=0$ and $p=1$.

The inviscid terms in Eq. (1) are approximated by the modified third-order upwind HLLEW scheme of Obayashi and Guruswamy. ${ }^{14}$ For the isentropic flow, the scheme results in the standard upwindbiased flux-difference splitting scheme of Roe, and as the jump in entropy becomes large in the flow, the scheme turns into the standard HLLEW scheme. The thin-layer viscous term in Eq. (1) is discretized by second-order central difference.

For multiblock-grid applications, the Navier-Stokes equations are solved in each block separately. To calculate the convective and viscous fluxes in the block boundary, data communication is 